\title{
Fully discrete FEM-BEM method for a class of exterior nonlinear parabolic-elliptic problems in 2D
}

\author{
María González*
}

June 20, 2005

\begin{abstract}
We considered a nonlinear parabolic equation in a bounded domain of $\mathbb{R}^{2}$ coupled with the Laplace equation in the corresponding exterior region. This kind of problems appears in the modelling of quasi-stationary electromagnetic fields. We chose a regular artificial boundary containing the nonlinear region in its interior. Then, we applied a symmetric FEM-BEM coupling procedure including a parameterization of the artificial boundary. We used the backward Euler method for the time discretization and an exact triangulation of the finite element domain. Assuming that the nonlinear operator is strongly monotone and Lipschitz-continuous, we proved convergence and obtained optimal error estimates for the solution of the discrete problem. Finally, we proposed a fully discrete scheme with quadrature formulas of low order and, under some additional conditions on the nonlinearity, proved that the order of convergence remains optimal.
\end{abstract}

Key words. boundary elements, finite elements, parabolic-elliptic problem

\section{Introduction}

In [16], R.C. MacCamy and M. Suri considered a linear parabolic-elliptic problem consisting of the heat equation in a bounded region of the plane coupled with the Laplace equation in the corresponding unbounded region. They used a finite element discretization for the spatial part of the heat equation and a boundary element method for the Laplace equation. They applied the standard method of C. Johnson and J.C. Nédélec [12] of coupling finite elements (FEM) and boundary elements (BEM) and proved, using a smooth coupling boundary, the convergence of their semidiscretized Galerkin scheme which lead to a system of ordinary differential equations (ode's) in time. An analysis of a scheme taking into account the discretization in time is not available and will be difficult since the stiffness matrix is neither symmetric nor positive definite.

*Departamento de Matemáticas, Universidade da Coruña, Campus de Elviña s/n 15071, A Coruña (Spain), mgtaboad@udc.es 
Later, M. Costabel, V.J. Ervin and E.P. Stephan [5] used the symmetric coupling method from M. Costabel [4] and H.D. Han [10] to solve the problem studied in [16]. They follow [16] in their convergence proof for the semidiscrete Galerkin scheme. After discretization in space, they obtained a system of ode's with a positive definite stiffness matrix. They applied the Crank-Nicolson method for its solution and proved convergence and theoretical error estimates for that fully discretized Galerkin scheme. More recently, P. Mund and E.P. Stephan [19] used the discontinuous Galerkin method to solve the system of ode's from [5] and proposed an adaptive algorithm based on an a posteriori error estimate that allows to choose the local mesh size $h$ and the time step independently.

In the symmetric method, most authors choose a polygonal curve as coupling boundary. However, in this case, we do not know how to control the effect of the use of quadratures on the convergence of the method. Moreover, the computation of some coefficients of the system is not easy due to the singularities of the integral operators. In [18], we presented a new version of the symmetric method based on a parameterization of the artificial boundary, which is a smooth curve, and the use of curved finite elements in the discretization. This procedure allows one to employ the techniques from G.C. Hsiao, P. Kopp and W.L. Wendland [11] to approximate the boundary terms by simple quadrature formulas and to study the effect of quadratures on convergence. This modified version of the symmetric method has also been used to solve the linear elasticity problem (cf.[17]).

In this work, we consider a model problem which consists of a nonlinear parabolic equation of second order in a bounded region of $\mathbb{R}^{2}$ and the Laplace equation in the corresponding exterior region; both equations are coupled by transmission conditions on the interface. This kind of problems appears in the modelling of two-dimensional nonlinear quasi-stationary electromagnetic fields (see [22] and the references therein). We show that the modified symmetric method described in [18] can be successfully applied to analyze this type of problems.

In Section 2 we describe the model problem and reduce it to a nonlinear parabolicelliptic problem in a bounded domain with nonlocal boundary conditions. In Section 3, we discretize the problem using the backward Euler method for the discretization in time and follow [18] for the discretization in space. We prove that, if the nonlinear operator is hemicontinuous and strongly monotone, the discrete problem is well-posed. In subsection 3.1 we prove that if the nonlinear operator is also bounded, then the discrete solutions converge to the unique solution of the continuous problem. Assuming that the nonlinear operator is also Lipschitz-continuous, we obtain in subsection 3.2 optimal error estimates. Finally, in Section 4 we propose a fully discrete scheme based on simple quadrature formulas and prove that, under some additional conditions, the order of convergence remains optimal.

Before describing the model problem, we introduce some notations that will be used throughout this paper. Let $X$ be a Banach space endowed with the norm $\|\cdot\|$ and let $T>0$ be a real number. We denote by $L^{2}(0, T ; X)$ the set of all functions $u:(0, T) \rightarrow X$ such that

$$
\|u\|_{L^{2}(0, T ; X)}^{2}:=\int_{0}^{T}\|u(t)\|^{2} d t<\infty
$$


The space $L^{2}(0, T ; X)$ is a Banach space endowed with the norm $\|\cdot\|_{L^{2}(0, T ; X)}$. Moreover, if $X$ is a Hilbert space with the inner product $(\cdot, \cdot)$, then $L^{2}(0, T ; X)$ is a Hilbert space with the inner product

$$
(u, v)_{L^{2}(0, T ; X)}:=\int_{0}^{T}(u(t), v(t)) d t
$$

The set of all continuous functions $u:[0, T] \rightarrow X$ is denoted $\mathcal{C}([0, T] ; X)$ and is a Banach space with the norm

$$
\|u\|_{\mathcal{C}([0, T] ; X)}:=\sup _{t \in[0, T]}\|u(t)\|
$$

For a detailed study of these spaces, we refer the reader to A. Kufner et al. [14].

We also use periodic Sobolev spaces. Let $\mathcal{C}^{\infty}$ denote the space of 1-periodic infinitely many differentiable real valued functions of a single variable. Given $g \in \mathcal{C}^{\infty}$, we define its Fourier coefficients

$$
\hat{g}(k):=\int_{0}^{1} g(s) e^{-2 k \pi i s} d s
$$

Then, for every $p \in \mathbb{R}$, we define the periodic Sobolev space $H^{p}$ as the completion of the space $\mathcal{C}^{\infty}$ with respect to the norm

$$
\|g\|_{p}:=\left(\sum_{k \in \mathbb{Z}}\left(1+|k|^{2}\right)^{p}|\hat{g}(k)|^{2}\right)^{1 / 2}
$$

It is well known that $H^{p}$ is a Hilbert space and that $H^{p} \subset H^{q}$ if $p>q$, the inclusion being dense and compact. Moreover, the $H^{0}$-inner product

$$
(\lambda, \mu):=\int_{0}^{1} \lambda(s) \mu(s) d s
$$

can be extended to represent the duality of $H^{-p}$ and $H^{p}$, for all $p$; we keep the same notation for the duality bracket. For more details, we refer to R. Kress [13].

Finally, throughout this paper, $C$, with or without subscripts, denotes a generic constant independent of the discretization parameters.

\section{The model problem}

Let $\Omega_{0}$ be a bounded domain of $\mathbb{R}^{2}$ with a Lipschitz boundary $\Gamma_{0}$, and denote $\Omega_{0}^{\prime}=$ $\mathbb{R}^{2} \backslash \bar{\Omega}_{0}$. We consider a simple closed curve $\Gamma_{1}$ containing the domain $\bar{\Omega}_{0}$ in its interior. We denote by $\Omega_{P}$ the annular region bounded by the curves $\Gamma_{0}$ and $\Gamma_{1}$, and by $\Omega_{E}$ the complementary of $\bar{\Omega}_{0} \cup \bar{\Omega}_{P}$ in $\mathbb{R}^{2}$.

Given $f \in L^{2}\left(0, T ; L^{2}\left(\Omega_{P}\right)\right), u_{0} \in L^{2}\left(\Omega_{P}\right)$ and the continuous nonlinear function 


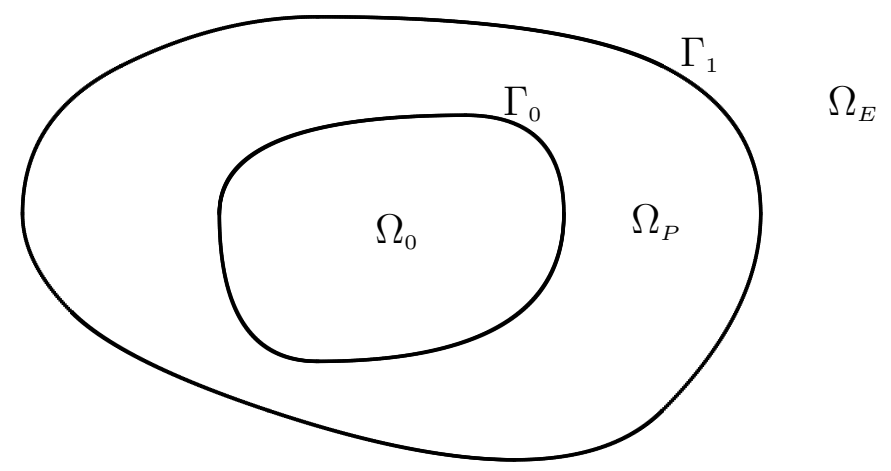

Figure 1: Domain of the model problem

$\mathbf{b}: \bar{\Omega}_{P} \times \mathbb{R}^{3} \rightarrow \mathbb{R}^{2}$, we look for a function $u:(0, T) \times \Omega_{0}^{\prime} \rightarrow \mathbb{R}$ such that for all $t \in(0, T)$,

$$
\begin{aligned}
\frac{\partial u}{\partial t}-\nabla \cdot \mathbf{b}(\cdot, u(t), \nabla u(t)) & =f(t) & & \text { in } \Omega_{P} \\
-\Delta u(t) & =0 & & \text { in } \Omega_{E} \\
u(t) & =0 & & \text { on } \Gamma_{0} \\
u_{P}(t) & =u_{E}(t) & & \text { on } \Gamma_{1} \\
\mathbf{b}\left(\cdot, u_{P}(t), \nabla u_{P}(t)\right) \cdot \mathbf{n}_{1} & =\frac{\partial u_{E}}{\partial \mathbf{n}_{1}}(t) & & \text { on } \Gamma_{1} \\
u(0) & =u_{0} & & \text { in } \Omega_{P} \\
u(t) & =\mathcal{O}(1) & & \text { as }|\mathbf{x}| \rightarrow+\infty
\end{aligned}
$$

where $\mathbf{n}_{1}$ is the unit outward normal vector to $\Gamma_{1}$, pointing from $\Omega_{P}$ to $\Omega_{E}$, and $u_{M}(t): \Gamma_{1} \rightarrow \mathbb{R}$ denotes the limit of $u(t)$ from $\Omega_{M}(M=E, P)$. We remark that the problem considered in [16] and [5] can be seen as a particular case of problem (1).

The basic idea of FEM-BEM methods is to introduce an artificial boundary dividing the domain of the problem in two regions, one bounded and another one unbounded, in such a way that the problem is linear and homogeneous with constant coefficients in the last. We consider a simple closed curve $\Gamma$ of class $\mathcal{C}^{\infty}$ containing the domain $\bar{\Omega}_{0} \cup \bar{\Omega}_{P}$ in its interior. The curve $\Gamma$ divides the domain $\Omega_{E}$ in two regions: a bounded one, which we denote $\Omega^{-}$, and an unbounded one, denoted $\Omega^{+}$. Then, problem (1) is equivalent to a transmission problem consisting, for each $t \in(0, T)$, of a nonlinear parabolic-elliptic problem posed in the bounded domain $\Omega:=\Omega_{P} \cup \Gamma_{1} \cup \Omega^{-}$:

$$
\begin{aligned}
\frac{\partial u}{\partial t}-\nabla \cdot \mathbf{b}(\cdot, u(t), \nabla u(t)) & =f(t) & & \text { in } \Omega_{P} \\
-\Delta u(t) & =0 & & \text { in } \Omega^{-} \\
u(t) & =0 & & \text { on } \Gamma_{0} \\
u_{P}(t) & =u_{E}(t) & & \text { on } \Gamma_{1} \\
\mathbf{b}\left(\cdot, u_{P}(t), \nabla u_{P}(t)\right) \cdot \mathbf{n}_{1} & =\frac{\partial u_{E}}{\partial \mathbf{n}_{1}}(t) & & \text { on } \Gamma_{1} \\
u(0) & =u_{0} & & \text { in } \Omega_{P}
\end{aligned}
$$




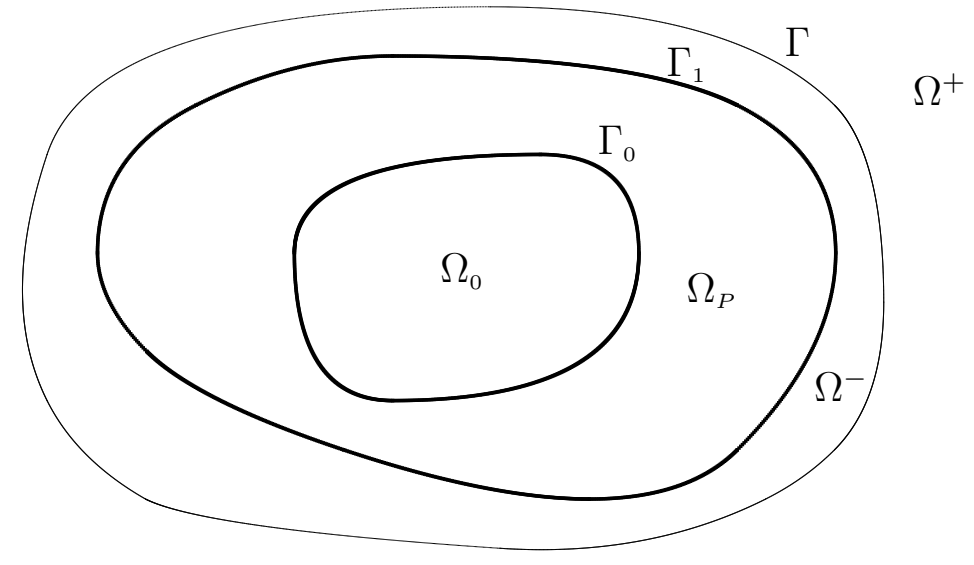

Figure 2: Domain of the transmission problem

and an elliptic problem, linear and homogeneous, posed in the unbounded region $\Omega^{+}$:

$$
\begin{array}{rll}
-\Delta u(t) & =0 & \text { in } \Omega^{+} \\
u(t) & =\mathcal{O}(1) & \text { as }|\mathbf{x}| \rightarrow+\infty
\end{array}
$$

Both problems are coupled by transmission conditions on $\Gamma$ :

$$
u^{-}(t)=u^{+}(t) \quad \frac{\partial u^{-}}{\partial \mathbf{n}}(t)=\frac{\partial u^{+}}{\partial \mathbf{n}}(t)
$$

Here, $\mathbf{n}$ is the unit normal vector to $\Gamma$, pointing from $\Omega^{-}$to $\Omega^{+}$, and $u^{ \pm}(t)$ is the limit of $u(t)$ on $\Gamma$ from $\Omega^{ \pm}$.

We remark that the boundary $\Gamma_{1}$ could be chosen as the coupling boundary if it is sufficiently smooth. However, this boundary is given by the problem and it is not necessarily smooth. This is the reason why we introduce the artificial boundary $\Gamma$.

In what follows, we suppose that there exists a constant $C>0$ such that

$$
|\mathbf{b}(\mathbf{x}, \boldsymbol{\alpha})| \leq C(1+|\boldsymbol{\alpha}|) \quad \forall \mathbf{x} \in \bar{\Omega}_{P} \quad \forall \boldsymbol{\alpha} \in \mathbb{R}^{3}
$$

where $|\cdot|$ stands for the Euclidean norm. We consider the nonlinear form $a: H^{1}(\Omega) \times$ $H^{1}(\Omega) \rightarrow \mathbb{R}$ defined by

$$
a(u, v):=a_{P}(u, v)+a_{E}(u, v) \quad \forall u, v \in H^{1}(\Omega)
$$

where

$$
a_{P}(u, v):=\int_{\Omega_{P}} \mathbf{b}(\cdot, u, \nabla u) \cdot \nabla v \quad \text { and } \quad a_{E}(u, v):=\int_{\Omega^{-}} \nabla u \cdot \nabla v
$$

Condition (5) ensures that $a(\cdot, \cdot)$ is well defined and bounded in $H^{1}(\Omega)$ (cf. $[22,9]$ ).

We see that the solution of problem (2) satisfies the following variational formulation:

$$
\int_{\Omega_{P}} \frac{\partial u}{\partial t}(t) v+a(u(t), v)-\int_{\Gamma} \frac{\partial u^{-}}{\partial \mathbf{n}}(t) v^{-}=\int_{\Omega_{P}} f(t) v \quad \forall v \in H_{\Gamma_{0}}^{1}(\Omega) \quad \text { a.e. } t \in(0, T)
$$


where $H_{\Gamma_{0}}^{1}(\Omega):=\left\{v \in H^{1}(\Omega) ;\left.v\right|_{\Gamma_{0}}=0\right\}$.

On the other hand, for every $t \in(0, T)$, the solution of problem (3) can be computed in every point of $\Omega^{+}$from the representation formula

$$
u(t, \mathbf{x})=-\frac{1}{2 \pi} \int_{\Gamma}\left(u(t, \mathbf{y}) \frac{\partial}{\partial \mathbf{n}_{\mathbf{y}}} \log |\mathbf{x}-\mathbf{y}|-\log |\mathbf{x}-\mathbf{y}| \frac{\partial}{\partial \mathbf{n}_{\mathbf{y}}} u(t, \mathbf{y})\right) d \sigma_{\mathbf{y}}+c_{u}
$$

where $c_{u}$ is a constant depending on $u$.

The symmetric FEM-BEM method consists in coupling (6) with two integral identities on $\Gamma$ deduced from (7) that relate the trace of $u$ and its normal derivative $\frac{\partial u}{\partial \mathbf{n}}$ (cf. [4, 10]); the coupling is possible thanks to the transmission conditions (4). This method, unlike the one of Johnson and Nédélec [12], allows to choose as artificial boundary a polygonal curve. However, this choice entails some difficulties due to the singularities of the boundary terms (cf. $[4,5,9]$ ).

Here, we follow [18] and assumed that the artificial boundary $\Gamma$ is smooth. Then, we consider a regular 1-periodic parameterization of $\Gamma, \boldsymbol{x}: \mathbb{R} \rightarrow \mathbb{R}^{2}$. Then we write all the integrals on the artificial boundary as integrals on $[0,1]$. In this way, we deduce the following variational formulation of problem (1): Find $u \in L^{2}\left(0, T ; H_{\Gamma_{0}}^{1}(\Omega)\right) \cap$ $\mathcal{C}\left([0, T] ; L^{2}\left(\Omega_{P}\right)\right)$ and $\xi \in L^{2}\left(0, T ; H_{0}^{-1 / 2}\right)$ such that $u(0)=u_{0}$ in $L^{2}\left(\Omega_{P}\right)$ and for all $v \in H_{\Gamma_{0}}^{1}(\Omega), \eta \in H_{0}^{-1 / 2}$ and a.e. $t \in(0, T)$

$$
\begin{aligned}
\frac{d}{d t}(u(t), v)_{L^{2}\left(\Omega_{P}\right)}+a(u(t), v)+d(u(t), v)-c(v, \xi(t)) & =(f(t), v)_{L^{2}\left(\Omega_{P}\right)} \\
c(u(t), \eta)+b(\xi(t), \eta) & =0
\end{aligned}
$$

where $\xi(t):=\frac{\partial u}{\partial \mathbf{n}}(\boldsymbol{x}(t))\left|\boldsymbol{x}^{\prime}(t)\right|$ is the scaled normal derivative and

$$
H_{0}^{-1 / 2}:=\left\{\eta \in H^{-1 / 2} ;(\eta, 1)=0\right\}
$$

We recall that the zero-mean condition on $\xi$ is a consequence of $(7)$ and the asymptotic behaviour of $u$ at infinity. The bilinear forms $b(\cdot, \cdot), d(\cdot, \cdot)$ and $c(\cdot, \cdot)$ are defined as follows, for all $\xi, \eta \in H^{-1 / 2}$ and $u, v \in H^{1}(\Omega)$,

$$
b(\xi, \eta):=(\eta, \mathcal{V} \xi) \quad d(u, v):=b\left(\gamma(u)^{\prime}, \gamma(v)^{\prime}\right) \quad c(v, \eta):=\left(\eta,\left(\frac{1}{2} \mathcal{I}-\mathcal{K}\right) \gamma(v)\right)
$$

Here, $\gamma: H^{1}(\Omega) \rightarrow H^{1 / 2}$ is the parameterized trace, and $\mathcal{V}: H^{-1 / 2} \rightarrow H^{1 / 2}$ and $\mathcal{K}: H^{1 / 2} \rightarrow H^{1 / 2}$ are parameterized versions of the simple and double layer operators, respectively. Next we recall their properties, which are consequence of the properties of the classical simple and double layer operators (cf. [13]).

Lemma 1. The linear operator $\mathcal{V}: H^{-1 / 2} \rightarrow H^{1 / 2}$ is continuous. Moreover, there exists a constant $\beta>0$ such that

$$
(\eta, \mathcal{V} \eta) \geq \beta\|\eta\|_{-1 / 2}^{2} \quad \forall \eta \in H_{0}^{-1 / 2}
$$

The operator $\mathcal{K}: H^{1 / 2} \rightarrow H^{1 / 2}$ is linear and compact. 
We recall that in the two boundary integral approach from Costabel [4] and Han [10], the compactness of the double layer operator is no longer crucial as it is in the approach of Johnson and Nédélec [12]. This fact justify the use of this method in elasticity (cf. $[6,2,17])$, where the double layer operator is never compact, even if the boundary is a smooth curve.

Now, we write the variational formulation (8) in an equivalent form, easier to analyze. For this purpose, we introduce the product space $M:=H_{\Gamma_{0}}^{1}(\Omega) \times H_{0}^{-1 / 2}$ and the form $A: M \times M \rightarrow \mathbb{R}$ given by

$$
A(\hat{u}, \hat{v}):=a(u, v)+B(\hat{u}, \hat{v}) \quad \forall \hat{u}:=(u, \xi), \hat{v}:=(v, \eta) \in M
$$

where $B(\cdot, \cdot)$ is the bilinear form defined by

$$
B(\hat{u}, \hat{v}):=d(u, v)-c(v, \xi)+c(u, \eta)+b(\xi, \eta) \quad \forall \hat{u}, \hat{v} \in M
$$

Then, problem (8) is equivalent to: Find $\hat{u}:=(u, \xi) \in L^{2}(0, T ; M), u \in \mathcal{C}\left([0, T] ; L^{2}\left(\Omega_{P}\right)\right)$ such that $u(0)=u_{0}$ in $L^{2}\left(\Omega_{P}\right)$ and a.e. $t \in(0, T)$ satisfies

$$
\frac{d}{d t}(u(t), v)_{L^{2}\left(\Omega_{P}\right)}+A(\hat{u}(t), \hat{v})=(f(t), v)_{L^{2}\left(\Omega_{P}\right)} \quad \forall \hat{v} \in M
$$

The analysis of problem (9) is based on the properties of the nonlinear function $\mathbf{b}$. In the rest of the paper, we assume that, besides (5), the derivatives $\frac{\partial b_{i}}{\partial \alpha_{j}}(i=1,2 ; j=0,1,2)$ are continuous in $\bar{\Omega}_{P} \times \mathbb{R}^{3}$ and there exist constants $\delta>0$ and $C>0$ such that for all $\mathbf{x} \in \bar{\Omega}_{P}$ and $\boldsymbol{\alpha} \in \mathbb{R}^{3}$,

$$
\sum_{i=1}^{2} \sum_{j=0}^{2} \frac{\partial b_{i}}{\partial \alpha_{j}}(\mathbf{x}, \boldsymbol{\alpha}) \beta_{i} \beta_{j} \geq \delta\left(\beta_{1}^{2}+\beta_{2}^{2}\right) \quad \forall \boldsymbol{\beta} \in \mathbb{R}^{3}
$$

and

$$
\left|\frac{\partial b_{i}}{\partial \alpha_{j}}(\mathbf{x}, \boldsymbol{\alpha})\right| \leq C
$$

Lemma 2. Under the assumptions made on $\mathbf{b}$, the form $A(\cdot, \cdot)$ is bounded, hemicontinuous, strongly monotone and Lipschitz-continuous in $M$.

Proof. In Theorem 32.6 of [22] it is proved that if conditions (5), (10) and (11) hold, then $a(\cdot, \cdot)$ is bounded and Lipschitz-continuous in $H^{1}(\Omega)$ and strongly monotone in $H_{\Gamma_{0}}^{1}(\Omega)$. Moreover, because the theorem of continuous dependence of the integral with respect to a parameter, $a(\cdot, \cdot)$ is also hemicontinuous (cf. Theorem 2.1.6 in [14]). On the other hand, due to Lemma 1 and the continuity of $\gamma: H^{1}(\Omega) \rightarrow H^{1 / 2}$ and $\frac{d}{d s}: H^{1 / 2} \rightarrow H_{0}^{-1 / 2}$, the bilinear form $B: M \times M \rightarrow \mathbb{R}$ is continuous in $M$. We also have that

$$
B(\hat{v}, \hat{v})=b\left(\gamma(v)^{\prime}, \gamma(v)^{\prime}\right)+b(\eta, \eta) \geq \beta\|\eta\|_{-1 / 2}^{2} \quad \forall \hat{v} \in M
$$

Therefore, $A(\cdot, \cdot)$ inherits the properties of $a(\cdot, \cdot)$. 


\section{The discrete problem}

In this section we propose and analyze a discrete scheme to solve problem (9). We use the backward Euler method for the discretization in time and follow [18] for the discretization in space. For simplicity, in what follows we suppose that the boundaries $\Gamma_{0}$ and $\Gamma_{1}$ are polygonal curves. We also assume that $f \in \mathcal{C}\left([0, T] ; L^{2}\left(\Omega_{P}\right)\right)$.

Given two positive integer numbers, $N_{0}$ and $N_{1}$, we consider a uniform partition of the interval $[0, T]$ of step $\Delta t:=T / N_{0}, t_{n}:=n \Delta t\left(n=0, \ldots, N_{0}\right)$, and a uniform partition of the real line of step $h:=1 / N_{1}, z_{i}:=i h(i \in \mathbb{Z})$. Then, let $\left(\tau_{h}\right)_{h}$ be a regular family of exact triangulations of the domain $\bar{\Omega}$ (cf. [22, 23, 24]). We suppose that $\tau_{h}$ is compatible with the interface $\Gamma_{1}$, and denote by $\tau_{h, E}$ and $\tau_{h, P}$ the triangulations of $\bar{\Omega}^{-}$and $\bar{\Omega}_{P}$ determined by $\tau_{h}$, respectively. We denote by $\sigma_{h}$ the set of vertices of the triangulation $\tau_{h}$ and suppose that $\sigma_{h} \cap \Gamma=\left\{\boldsymbol{x}\left(z_{i}\right)\right\}_{i=0}^{N_{1}}$.

To approximate the unknown $u(t)$, we consider continuous piecewise affine functions in $\bar{\Omega}$ vanishing on $\Gamma_{0}$, that is, we define the space

$$
V_{h}:=\left\{v_{h} \in \mathcal{C}(\bar{\Omega}) ;\left.v_{h}\right|_{T} \in \mathcal{P}_{1}(T) \quad \forall T \in \tau_{h} \text { and }\left.v_{h}\right|_{\Gamma_{0}}=0\right\}
$$

To approximate the scaled normal derivative $\xi$, we define the space

$$
H_{h}:=\left\{\eta_{h} \in H^{0} ; \eta_{i}:=\left.\eta_{h}\right|_{\left(z_{i-1}, z_{i}\right)} \in \mathbb{R} \quad \forall i \in \mathbb{Z} \quad \text { and } \quad\left(\eta_{h}, 1\right)=0\right\}
$$

Lastly, we define the product space $M_{h}:=V_{h} \times H_{h}$.

We will assume that $\left(u_{0, h}\right)_{h}$ is a sequence of continuous functions in $\bar{\Omega}_{P}$ such that $\left(u_{0, h}\right)_{\mid T} \in \mathcal{P}_{1}(T) \forall T \in \tau_{h, P}$ and that $u_{0, h} \rightarrow u_{0}$ in $L^{2}\left(\Omega_{P}\right)$. Then, we consider the following discrete scheme: Find $\hat{u}_{h}^{n}:=\left(u_{h}^{n}, \xi_{h}^{n}\right) \in M_{h}\left(n=1, \ldots, N_{0}\right)$ such that

$$
\left(u_{h}^{n}-u_{h}^{n-1}, v_{h}\right)_{L^{2}\left(\Omega_{P}\right)}+\Delta t A\left(\hat{u}_{h}^{n}, \hat{v}_{h}\right)=\Delta t\left(f\left(t_{n}\right), v_{h}\right)_{L^{2}\left(\Omega_{P}\right)} \quad \forall \hat{v}_{h} \in M_{h}
$$

where $u_{h}^{0}:=u_{0, h}$. Here, $\hat{u}_{h}^{n}$ is expected to be an approximation of $\hat{u}\left(t_{n}\right)$ and the backward difference $\left.\Delta t^{-1}\left(u_{h}^{n}-u_{h}^{n-1}\right)\right|_{\Omega_{P}}$ is an approximation of $\left.u^{\prime}\left(t_{n}\right)\right|_{\Omega_{P}}$, for $n=$ $1, \ldots, N_{0}$.

Theorem 3. Assume that $\mathbf{b}$ satisfies (5) and (10). Then, problem (12) has a unique solution for $h$ sufficiently small.

Proof. Let $\left\{\phi_{i}\right\}_{i=1}^{d_{1}}$ and $\left\{\psi_{k}\right\}_{k=1}^{d_{2}}$ be basis of $V_{h}$ and $H_{h}$, respectively, and denote $D:=$ $d_{1}+d_{2}$. We consider the product basis of $M_{h},\left\{\widehat{\Psi}_{d}\right\}_{d=1}^{D}$, where for $d=1, \ldots, D$,

$$
\widehat{\Psi}_{d}:= \begin{cases}\left(\phi_{d}, 0\right) & \text { if } d \in\left\{1, \ldots, d_{1}\right\} \\ \left(0, \psi_{d-d_{1}}\right) & \text { if } d \in\left\{d_{1}+1, \ldots, D\right\}\end{cases}
$$

Then, for $n=1, \ldots, N_{0}, \hat{u}_{h}^{n}=\sum_{d=1}^{D} u_{d}^{n} \widehat{\Psi}_{d}$ and problem (12) is equivalent to $N_{0}$ systems of nonlinear equations:

$$
\mathbf{f}\left(\mathbf{u}^{n}\right)=\mathbf{g}^{n} \quad\left(n=1, \ldots, N_{0}\right)
$$

where

$$
\mathrm{f}_{i}(\mathbf{u}):= \begin{cases}\sum_{j=1}^{d_{1}} u_{j}\left(\phi_{j}, \phi_{i}\right)_{L^{2}\left(\Omega_{P}\right)}+\Delta t A\left(\sum_{j=1}^{D} u_{j} \widehat{\Psi}_{j}, \widehat{\Psi}_{i}\right) & \text { if } i \in\left\{1, \ldots, d_{1}\right\} \\ \Delta t A\left(\sum_{j=1}^{D} u_{j} \widehat{\Psi}_{j}, \widehat{\Psi}_{i}\right) & \text { if } i \in\left\{d_{1}+1, \ldots, D\right\}\end{cases}
$$


and $\mathrm{g}_{i}^{n}:=\left(u_{h}^{n-1}, \phi_{i}\right)_{L^{2}\left(\Omega_{P}\right)}+\Delta t\left(f\left(t_{n}\right), \phi_{i}\right)_{L^{2}\left(\Omega_{P}\right)}$ if $i \in\left\{1, \ldots, d_{1}\right\}$ and $\mathrm{g}_{i}^{n}:=0$ otherwise.

By virtue of Lemma $2, A(\cdot, \cdot)$ is hemicontinuous and strongly monotone. Using these properties, it is easy to prove that $\mathbf{f}$ is hemicontinuous, strongly monotone and coercive. Then, applying Theorems 27.1 and 27.3 in J. Oden [20], we deduce that each of the systems (13) has a unique solution.

We remark that Theorem 3 is also true under weaker conditions on the coefficients of the nonlinear equation. In fact, (10) can be substituted by certain conditions so that $A(\cdot, \cdot)$ is monotone and coercive in $M$. These properties are sufficient to deduce that the function $\mathbf{f}$ in Theorem 3 is strongly monotone and coercive (cf. [20]).

\subsection{Existence, uniqueness and convergence}

In this subsection, we prove the existence and uniqueness of a solution to the continuous problem (9), and a convergence result in $L^{2}(0, T ; M)$. For this purpose, we use the a priori error estimate given in Lemma 4 below. In what follows, given any function $\phi \in \mathcal{C}([0, T])$, we denote by $\phi_{e}$ the piecewise constant function

$$
\phi_{e}(t):= \begin{cases}\phi\left(t_{n}\right) & \text { if } t \in\left(t_{n-1}, t_{n}\right]\left(n=1, \ldots, N_{0}\right) \\ \phi\left(t_{1}\right) & \text { if } t=0\end{cases}
$$

Lemma 4. If b satisfies (5) and (10), then, for $\Delta t$ sufficiently small, there exists a constant $C>0$, independent of $\Delta t$ and $h$, such that

$$
\left\|u_{h, e}(T)\right\|_{L^{2}\left(\Omega_{P}\right)}+\left\|\hat{u}_{h, e}\right\|_{L^{2}(0, T ; M)} \leq C
$$

Proof. The proof of this result is standard. We take $\hat{v}_{h}=\hat{u}_{h}^{n}$ in equation (12), sum up from $n=1$ to $n=N_{0}$ and remark that

$$
\sum_{n=1}^{N_{0}}\left(u_{h}^{n}-u_{h}^{n-1}, u_{h}^{n}\right)_{L^{2}\left(\Omega_{P}\right)}=\frac{1}{2} \sum_{n=1}^{N_{0}}\left\|u_{h}^{n}-u_{h}^{n-1}\right\|_{L^{2}\left(\Omega_{P}\right)}^{2}+\frac{1}{2}\left\|u_{h}^{N_{0}}\right\|_{L^{2}\left(\Omega_{P}\right)}^{2}-\frac{1}{2}\left\|u_{0, h}\right\|_{L^{2}\left(\Omega_{P}\right)}^{2}
$$

Then, using that the sequence $\left(u_{0, h}\right)_{h}$ is bounded and the strong monotonicity of $A(\cdot, \cdot)$, we have that

$$
\frac{1}{2}\left\|u_{h}^{N_{0}}\right\|_{L^{2}\left(\Omega_{P}\right)}^{2}+\hat{\alpha} \sum_{n=1}^{N_{0}}\left\|\hat{u}_{h}^{n}\right\|_{M}^{2} \leq C+\Delta t \sum_{n=1}^{N_{0}}\left\|f\left(t_{n}\right)\right\|_{L^{2}\left(\Omega_{P}\right)}\left\|\hat{u}_{h}^{n}\right\|_{M}
$$

where $\hat{\alpha}$ is the monotonicity constant of $A(\cdot, \cdot)$. The result follows applying the inequality $a b \leq \frac{a^{2}}{2 \epsilon}+\frac{\epsilon b^{2}}{2}$ with $a=\left\|f\left(t_{n}\right)\right\|_{L^{2}\left(\Omega_{P}\right)}, b=\left\|\hat{u}_{h}^{n}\right\|_{M}$ and $\epsilon=\hat{\alpha}$.

We will also use the following auxiliary result, which can be verified easily (cf. Lemma 46.2 in [22]).

Lemma 5. Let $\phi \in \mathcal{C}^{\infty}([0, T])$. Then there exist positive constants, $C_{1}$ and $C_{2}$, depending on the function $\phi$, such that

$$
\left\|\phi_{e}-\phi\right\|_{L^{2}(0, T)} \leq C_{1} \Delta t \quad\left\|\tilde{\phi}_{e}-\phi^{\prime}\right\|_{L^{2}(0, T)} \leq C_{2} \Delta t^{1 / 2}
$$


where

$$
\tilde{\phi}_{e}(t):= \begin{cases}\frac{\phi\left(t_{n+1}\right)-\phi\left(t_{n}\right)}{\Delta t} & \text { if } t \in\left(t_{n-1}, t_{n}\right]\left(n=1, \ldots, N_{0}\right) \\ \frac{\phi\left(t_{2}\right)-\phi\left(t_{1}\right)}{\Delta t} & \text { if } t=0\end{cases}
$$

with $\phi\left(t_{N_{0}+1}\right):=\phi(T)$.

The convergence of the approximate solutions to parabolic-elliptic problems obtained by finite element discretizations was first studied by M. Zlámal [25] and later by A. Ženíšek [22]. The proof given here follows essentially that of [22], but presents some simplifications because in [22], the author looks for an exact solution such that its derivative in $\Omega_{P}$ belongs to $L^{2}\left(0, T ; V_{P}^{\prime}\right)$, where $V_{P}^{\prime}$ is the dual space of $V_{P}=\{v \in$ $\left.H^{1}\left(\Omega_{P}\right): v_{\mid \Gamma_{0}}=0\right\}$.

Theorem 6. If $\mathbf{b}$ satisfies (5) and (10), then problem (9) has a unique solution $\hat{u}:=$ $(u, \xi)$ and the sequence $\left(\hat{u}_{h, e}\right)_{h}$ converges to $\hat{u}$ in $L^{2}(0, T ; M)$ as $h \rightarrow 0$.

Proof. We give a sketch of the proof. Firstly, since $L^{2}\left(\Omega_{P}\right)$ and $L^{2}(0, T ; M)$ are reflexive spaces, Lemma 4 implies that there exist subsequences $\left(u_{h^{\prime}, e}(T)\right)_{h^{\prime}}$ from $\left(u_{h, e}(T)\right)_{h}$ and $\left(\hat{u}_{h^{\prime}, e}\right)_{h^{\prime}}$ from $\left(\hat{u}_{h, e}\right)_{h}$ converging weakly in $L^{2}\left(\Omega_{P}\right)$ and in $L^{2}(0, T ; M)$, respectively; that is, there exist functions $g \in L^{2}\left(\Omega_{P}\right)$ and $\hat{u}:=(u, \xi) \in L^{2}(0, T ; M)$ such that

$$
\begin{gathered}
\left(u_{h^{\prime}, e}(T), v\right)_{L^{2}\left(\Omega_{P}\right)} \rightarrow(g, v)_{L^{2}\left(\Omega_{P}\right)} \quad \forall v \in L^{2}\left(\Omega_{P}\right) \\
\left(\hat{u}_{h^{\prime}, e}, \hat{v}\right)_{L^{2}(0, T ; M)} \rightarrow(\hat{u}, \hat{v})_{L^{2}(0, T ; M)} \quad \forall \hat{v} \in L^{2}(0, T ; M)
\end{gathered}
$$

Now, let $\hat{w}:=(w, \zeta) \in M$ and $\phi \in \mathcal{C}^{\infty}([0, T])$. We consider a sequence $\left(\hat{w}_{h}\right)_{h}$, $\hat{w}_{h} \in M_{h}$, such that $\hat{w}_{h} \rightarrow \hat{w}$ in $M$. Taking $\hat{v}_{h}=\hat{w}_{h} \phi\left(t_{n}\right)$ in (12) and summing by parts, we deduce that

$$
\begin{gathered}
\int_{0}^{T}\left(f_{e}(t), w_{h}\right) \phi_{e}(t) d t=\left(u_{h, e}(T), w_{h}\right) \phi(T)-\left(u_{0, h}, w_{h}\right) \phi(\Delta t) \\
\quad+\int_{0}^{T} A\left(\hat{u}_{h, e}(t), \hat{w}_{h}\right) \phi_{e}(t) d t-\int_{0}^{T}\left(u_{h, e}(t), w_{h}\right) \tilde{\phi}_{e}(t) d t
\end{gathered}
$$

where, for brevity, we denoted $(\cdot, \cdot)$ the inner product of $L^{2}\left(\Omega_{P}\right)$.

Since $A(\cdot, \cdot)$ is nonlinear, we cannot pass to the limit in (16) directly. Then, for each $h^{\prime}$ and each $t \in[0, T]$, we consider the functional $\chi_{h^{\prime}}(t) \in M^{\prime}$ defined by

$$
\left\langle\chi_{h^{\prime}}(t), \hat{v}\right\rangle:=A\left(\hat{u}_{h^{\prime}, e}(t), \hat{v}\right) \quad \forall \hat{v} \in M
$$

where $\langle\cdot, \cdot\rangle$ denotes the duality pairing between $M^{\prime}$ and $M$. Using the properties of $A(\cdot, \cdot)$, it is easy to prove that the sequence $\left(\chi_{h^{\prime}}\right)_{h^{\prime}}$ is bounded in $L^{2}\left(0, T ; M^{\prime}\right)$, which is a reflexive space. Therefore, there exists an element $\chi \in L^{2}\left(0, T ; M^{\prime}\right)$ and a subsequence of $\left(\chi_{h^{\prime}}\right)_{h^{\prime}}$, denoted the same, that converges weakly to $\chi$ in $L^{2}\left(0, T ; M^{\prime}\right)$.

Now, we can pass to the limit in (16) as $h^{\prime} \rightarrow 0$. Using the Cauchy-Schwarz inequality, Lemma 5, (14), (15) and the convergence of sequences $\left(u_{0, h}\right)_{h},\left(\hat{w}_{h}\right)_{h}$ and 
$\left(\chi_{h^{\prime}}\right)_{h^{\prime}}$, we obtain:

$$
\begin{gathered}
\int_{0}^{T}(f(t), w)_{L^{2}\left(\Omega_{P}\right)} \phi(t) d t=(g, w)_{L^{2}\left(\Omega_{P}\right)} \phi(T)-\left(u_{0}, w\right)_{L^{2}\left(\Omega_{P}\right)} \phi(0) \\
+\int_{0}^{T}\langle\chi(t), \hat{w}\rangle \phi(t) d t-\int_{0}^{T}(u(t), w)_{L^{2}\left(\Omega_{P}\right)} \phi^{\prime}(t) d t
\end{gathered}
$$

Taking $\phi \in \mathcal{C}_{0}^{\infty}(0, T)$ and integrating by parts, we deduce that

$$
\frac{d}{d t}(u(t), w)_{L^{2}\left(\Omega_{P}\right)}=(f(t), w)_{L^{2}\left(\Omega_{P}\right)}-\langle\chi(t), \hat{w}\rangle \quad \text { a.e. } t \in(0, T) \quad \forall \hat{w} \in M
$$

Now, thanks to the regularity of $u, f$ and $\chi$, the mapping $t \mapsto(u(t), w)_{L^{2}\left(\Omega_{P}\right)}$ belongs to $H^{1}(0, T)$. Therefore, we can identify it with a function in $\mathcal{C}[0, T]$, so $u \in$ $\mathcal{C}\left([0, T] ; L^{2}\left(\Omega_{P}\right)\right)$. Then, from (19) we deduce that for every $\hat{w} \in M$ and for every $t \in[0, T]$

$$
(u(t), w)_{L^{2}\left(\Omega_{P}\right)}=(u(0), w)_{L^{2}\left(\Omega_{P}\right)}+\int_{0}^{t}\left\{(f(s), w)_{L^{2}\left(\Omega_{P}\right)}-\langle\chi(s), \hat{w}\rangle\right\} d s
$$

If we consider again relation (18) with $\phi \in \mathcal{C}^{\infty}([0, T])$ such that $\phi(0)=1$ and $\phi(T)=0$, and integrate by parts, we obtain that $u(0)=u_{0}$ in $L^{2}\left(\Omega_{P}\right)$. Similarly, we deduce that $g=u(T)$ in $L^{2}\left(\Omega_{P}\right)$.

Next we prove that $\hat{u}$ satisfies equation (9) and that the subsequence $\left(\hat{u}_{h^{\prime}, e}\right)_{h^{\prime}}$ converges to $\hat{u}$ in $L^{2}(0, T ; M)$. We recall that under assumptions $(5)$ and $(10), A(\cdot, \cdot)$ is strongly monotone in $M$. Thus, for all $\hat{v} \in M$,

$$
\int_{0}^{T}\left(A\left(\hat{u}_{h^{\prime}, e}(t), \hat{u}_{h^{\prime}, e}(t)-\hat{v}\right)-A\left(\hat{v}, \hat{u}_{h^{\prime}, e}(t)-\hat{v}\right)\right) d t \geq \hat{\alpha}\left\|\hat{u}_{h^{\prime}, e}-\hat{v}\right\|_{L^{2}(0, T ; M)}^{2}
$$

On the other hand, proceeding as in the proof of Lemma 4, we deduce that

$$
\int_{0}^{T} A\left(\hat{u}_{h^{\prime}, e}(t), \hat{u}_{h^{\prime}, e}(t)\right) \leq \frac{1}{2}\left\|u_{0, h^{\prime}}\right\|_{L^{2}\left(\Omega_{P}\right)}^{2}-\frac{1}{2}\left\|u_{h^{\prime}, e}(T)\right\|_{L^{2}\left(\Omega_{P}\right)}^{2}+\int_{0}^{T}\left(f_{e}(t), u_{h^{\prime}, e}(t)\right)_{L^{2}\left(\Omega_{P}\right)}
$$

Then, taking into account definition (17), the weak convergence of $\left(\chi_{h^{\prime}}\right)_{h^{\prime}},(14)$ and (15), we deduce from (20) that for all $\hat{v} \in M$,

$$
\hat{\alpha} \limsup _{h^{\prime} \rightarrow 0}\left\|\hat{u}_{h^{\prime}, e}-\hat{v}\right\|_{L^{2}(0, T ; M)}^{2} \leq \int_{0}^{T}\langle\chi(t), \hat{u}(t)-\hat{v}\rangle d t-\int_{0}^{T} A(\hat{v}, \hat{u}(t)-\hat{v}) d t
$$

Now, given $\hat{w} \in L^{2}(0, T ; M)$ and $\theta \in(0,1)$, we take $\hat{v}=\hat{u}(t)-\theta \hat{w}(t)$ and pass to the limit in $(21)$ as $\theta \rightarrow 0$. Using that $A(\cdot, \cdot)$ is hemicontinuous and bounded, and applying the theorem of dominated convergence, we deduce, by linearity, that

$$
\int_{0}^{T}\langle\chi(t), \hat{w}(t)\rangle d t=\int_{0}^{T} A(\hat{u}(t), \hat{w}(t)) d t \quad \forall \hat{w} \in L^{2}(0, T ; M)
$$

Then, taking $\hat{v}=\hat{u}$ in $(21)$, we deduce that the subsequence $\left(\hat{u}_{h^{\prime}, e}\right)_{h^{\prime}}$ converges to $\hat{u}$ in $L^{2}(0, T ; M)$. Moreover, taking $\hat{w}(x, t)=\hat{v}(x) \phi(t)$ in $(22)$, with $\phi \in \mathcal{C}_{0}^{\infty}(0, T)$ and $\hat{v} \in M$, we have

$$
\langle\chi(t), \hat{v}\rangle=A(\hat{u}(t), \hat{v}) \quad \text { a.e. } t \in(0, T) \forall \hat{v} \in M
$$


Therefore, $\hat{u}$ satisfies equation (9) (see (19)).

Finally, the uniqueness of the solution and the convergence of the whole sequence $\left(\hat{u}_{h, e}\right)_{h}$ to $\hat{u}$ in $L^{2}(0, T ; M)$ can be proved using standard arguments.

Lemma 4 can be proved under weaker conditions on the function $\mathbf{b}$. In fact, it suffices the monotonicity and coercivity of $A(\cdot, \cdot)$ (cf. [22]). Therefore, from the proof of Theorem 6 it is clear that the existence and uniqueness of a solution to problem (9) can also be obtained under these conditions. Assumption (10) is only necessary to ensure the strong convergence of $\left(\hat{u}_{h, e}\right)_{h}$ to $\hat{u}$ in $L^{2}(0, T ; M)$.

\subsection{Error estimates}

In this subsection, we suppose that the solution $u$ of problem (9) has the regularity $L^{2}\left(0, T ; H^{1+\sigma}(\Omega)\right)$, with $\sigma \in[0,1)$, and we obtain error estimates in terms of the discretization parameters using standard techniques (cf. V. Thomée [21]). Instead of using the elliptic projection operator, that requires duality techniques, we use the interpolation operator defined by C. Bernardi [1] which is based on a local $L^{2}$-projection. This operator is a generalization of the one introduced by $\mathrm{Ph}$. Clément [3]. Then, given $u \in L^{1}(\Omega), \Pi_{h} u$ denotes the interpolate of $u$ in $V_{h}$ as defined in [1].

Lemma 7. If $w \in H_{\Gamma_{0}}^{1}(\Omega) \cap H^{1+\sigma}(\Omega)$, with $\sigma \in[0,1)$, then there exists a constant $C>0$, independent of $h$, such that

$$
\left\|w-\Pi_{h} w\right\|_{k, \Omega} \leq C h^{1+\sigma-k}\|w\|_{H^{1+\sigma}(\Omega)} \quad(k=0,1)
$$

Proof. It is well known that (cf. [1]), if $w \in H_{\Gamma_{0}}^{1}(\Omega) \cap H^{m}(\Omega)(m=1,2)$, then there exists a constant $C>0$, independent of $h$, such that

$$
\left\|w-\Pi_{h} w\right\|_{k, \Omega} \leq C h^{m-k}\|w\|_{m, \Omega} \quad(k=0,1)
$$

The result follows using the interpolation theory in Sobolev spaces (cf. [15]).

Theorem 8. Assume that $u \in \mathcal{C}\left((0, T] ; H^{1+\sigma}(\Omega)\right), u^{\prime} \in L^{2}\left(0, T ; H^{1}(\Omega)\right), u^{\prime \prime} \in L^{2}\left(0, T ; V_{P}^{\prime}\right)$, $u_{0} \in H^{1}\left(\Omega_{P}\right)$ and that there exists a constant $C$, independent of $h$, such that

$$
\left\|u_{0}-u_{0, h}\right\|_{L^{2}\left(\Omega_{P}\right)} \leq C h\left\|u_{0}\right\|_{H^{1}\left(\Omega_{P}\right)}
$$

Then, if $\mathbf{b}$ satisfies (5), (10) and (11), there exists a constant $C>0$, independent of $h$ and $\Delta t$, such that

$$
\left\|\hat{u}_{e}-\hat{u}_{h, e}\right\|_{L^{2}(0, T ; M)} \leq C\left(h^{\sigma}+\Delta t\right)
$$

Proof. Let $\pi_{h}: H^{0} \rightarrow H_{h}$ be the orthogonal projection operator in $H^{0}$ and consider the operator $\widehat{\Pi}_{h} \hat{v}:=\left(\Pi_{h} v, \pi_{h} \eta\right) \forall \hat{v}:=(v, \eta) \in L^{1}(\Omega) \times H^{0}$. For $n=1, \ldots, N_{0}$, we denote by $\hat{e}_{h}^{n}=\left(e_{h}^{n}, \epsilon_{h}^{n}\right):=\hat{u}_{h}^{n}-\widehat{\Pi}_{h} \hat{u}\left(t_{n}\right)$, that is, $e_{h}^{n}=u_{h}^{n}-\tilde{u}_{h}^{n}$, with $\tilde{u}_{h}^{n}:=\Pi_{h} u\left(t_{n}\right)$, and $\epsilon_{h}^{n}=\xi_{h}^{n}-\pi_{h} \xi\left(t_{n}\right)$. We choose $\tilde{u}_{h}^{0}:=u_{0, h}$ and put $e_{h}^{0}:=0$. We recall that, in the 
conditions of the theorem, $\hat{u}$ satisfies equation (9) for all $t \in(0, T]$. Since $\left(\hat{u}_{h}^{n}\right)_{n=1}^{N_{0}}$ is the solution to problem (12), we deduce that

$$
\begin{aligned}
\left(e_{h}^{n}-e_{h}^{n-1}, v_{h}\right)_{L^{2}\left(\Omega_{P}\right)} & +\Delta t\left(A\left(\hat{u}_{h}^{n}, \hat{v}_{h}\right)-A\left(\widehat{\Pi}_{h} \hat{u}\left(t_{n}\right), \hat{v}_{h}\right)\right) \\
& =\left(\Delta t u^{\prime}\left(t_{n}\right)-\left(u\left(t_{n}\right)-u\left(t_{n-1}\right)\right), v_{h}\right)_{L^{2}\left(\Omega_{P}\right)} \\
& +\Delta t\left(A\left(\hat{u}\left(t_{n}\right), \hat{v}_{h}\right)-A\left(\widehat{\Pi}_{h} \hat{u}\left(t_{n}\right), \hat{v}_{h}\right)\right) \\
& +\left(u\left(t_{n}\right)-u\left(t_{n-1}\right)-\left(\tilde{u}_{h}^{n}-\tilde{u}_{h}^{n-1}\right), v_{h}\right)_{L^{2}\left(\Omega_{P}\right)}
\end{aligned}
$$

Next, we estimate the terms appearing in the right hand side of (24). A Taylor expansion of first order in $V_{P}^{\prime}$ gives that

$$
\left(\Delta t u^{\prime}\left(t_{n}\right)-\left(u\left(t_{n}\right)-u\left(t_{n-1}\right)\right), v_{h}\right)_{L^{2}\left(\Omega_{P}\right)}=\left\langle\int_{t_{n-1}}^{t_{n}}\left(t_{n-1}-t\right) u^{\prime \prime}(t) d t, v_{h}\right\rangle_{P}
$$

where $\langle\cdot, \cdot\rangle_{P}$ denotes the duality pairing between $V_{P}^{\prime}$ and $V_{P}$. Then, using the CauchySchwarz inequality, we deduce that

$$
\left|\left(\Delta t u^{\prime}\left(t_{n}\right)-\left(u\left(t_{n}\right)-u\left(t_{n-1}\right)\right), v_{h}\right)_{L^{2}\left(\Omega_{P}\right)}\right| \leq \Delta t^{3 / 2}\left\|u^{\prime \prime}\right\|_{L^{2}\left(0, T ; V_{P}^{\prime}\right)}\left\|v_{h}\right\|_{L^{2}\left(\Omega_{P}\right)}
$$

On the other hand, if conditions (5) and (11) are satisfied, then $A(\cdot, \cdot)$ is Lipschitzcontinuous. Thus, applying Lemma 7 we obtain

$$
\left|\Delta t\left(A\left(\hat{u}\left(t_{n}\right), \hat{v}_{h}\right)-A\left(\widehat{\Pi}_{h} \hat{u}\left(t_{n}\right), \hat{v}_{h}\right)\right)\right| \leq C \Delta t h^{\sigma}\left\|u\left(t_{n}\right)\right\|_{H^{1+\sigma}(\Omega)}\left\|\hat{v}_{h}\right\|_{M}
$$

Finally, using Lemma 7 for $n=2, \ldots, N_{0}$, we deduce

$$
\begin{aligned}
\left|\left(u\left(t_{n}\right)-u\left(t_{n-1}\right)-\left(\tilde{u}_{h}^{n}-\tilde{u}_{h}^{n-1}\right), v_{h}\right)_{L^{2}\left(\Omega_{P}\right)}\right| & \leq C h\left\|u\left(t_{n}\right)-u\left(t_{n-1}\right)\right\|_{H^{1}(\Omega)}\left\|v_{h}\right\|_{L^{2}\left(\Omega_{P}\right)} \\
& \leq C \Delta t^{1 / 2} h\left\|u^{\prime}\right\|_{L^{2}\left(0, T ; H^{1}(\Omega)\right)}\left\|v_{h}\right\|_{L^{2}\left(\Omega_{P}\right)}
\end{aligned}
$$

If $n=1$, we apply Lemma 7 and inequality (23) to obtain

$$
\left|\left(u\left(t_{1}\right)-u_{0}-\left(\tilde{u}_{h}^{1}-\tilde{u}_{h}^{0}\right), v_{h}\right)_{L^{2}\left(\Omega_{P}\right)}\right| \leq C h\left(\left\|u\left(t_{1}\right)\right\|_{H^{1}(\Omega)}+\left\|u_{0}\right\|_{H^{1}\left(\Omega_{P}\right)}\right)\left\|v_{h}\right\|_{L^{2}\left(\Omega_{P}\right)}
$$

The result follows taking $\hat{v}_{h}=\hat{e}_{h}^{n}\left(n=1, \ldots, N_{0}\right)$ in (24), using the strong monotonicity of $A(\cdot, \cdot)$ and applying repeatedly inequality $a b \leq \frac{a^{2}}{2 \epsilon}+\frac{\epsilon b^{2}}{2}$.

\section{The effect of quadratures on convergence}

In practice, it is not possible to solve problem (12) directly since we cannot compute exactly all the coefficients of the system. We have to use quadrature formulas to approximate the integrals over the nonlinear region, the curved triangles and those corresponding to boundary terms. In this section we propose and analyze a fully discrete scheme that takes into account the use of quadrature formulas. 
To approximate the coefficients in which appear integrals on $\Omega$, we consider a quadrature formula on a reference triangle:

$$
\int_{\widehat{T}} \hat{\phi}(\hat{\mathbf{x}}) d \hat{\mathbf{x}} \simeq \widehat{Q}(\hat{\phi}):=\sum_{l=1}^{L} \hat{\omega}_{l} \hat{\phi}\left(\hat{\mathbf{a}}_{l}\right)
$$

and suppose that it is exact for constant functions. Then, on every triangle $T \in \tau_{h}$, we obtain the corresponding quadrature formula $Q_{T}$ through a change of variables.

We define the nonlinear form $a_{h}: V_{h} \times V_{h} \rightarrow \mathbb{R}$ as

$$
a_{h}\left(u_{h}, v_{h}\right):=a_{h, P}\left(u_{h}, v_{h}\right)+a_{h, E}\left(u_{h}, v_{h}\right) \quad \forall u_{h}, v_{h} \in V_{h}
$$

where

$$
a_{h, P}\left(u_{h}, v_{h}\right):=\sum_{T \in \tau_{h, P}} Q_{T}\left(\mathbf{b}\left(\cdot, u_{h}, \nabla u_{h}\right) \cdot \nabla v_{h}\right) \quad a_{h, E}\left(u_{h}, v_{h}\right):=\sum_{T \in \tau_{h, E}} Q_{T}\left(\nabla u_{h} \cdot \nabla v_{h}\right)
$$

We also consider a perturbation of $\left(f\left(t_{n}\right), v_{h}\right)_{L^{2}\left(\Omega_{P}\right)}$ :

$$
\left(f\left(t_{n}\right), v_{h}\right)_{h, P}:=\sum_{T \in \tau_{h, P}} Q_{T}\left(f\left(t_{n}\right) v_{h}\right) \quad \forall v_{h} \in V_{h}
$$

For the approximation of the boundary terms, we follow [18]. We consider a quadrature formula $\hat{\ell}_{2}(\cdot)$ on the unit square and assume that it is exact for polynomials of degree less than or equal to one. Next we use this quadrature to define perturbations $b_{h}(\cdot, \cdot), d_{h}(\cdot, \cdot)$ and $c_{h}(\cdot, \cdot)$ of the bilinear forms $b(\cdot, \cdot), d(\cdot, \cdot)$ and $c(\cdot, \cdot)$, respectively.

For $i=1, \ldots, N_{1}$, we denote by $\mu_{i}$ the 1 -periodic function given by

$$
\mu_{i}(s):= \begin{cases}1 & \text { if } s \in\left(z_{i-1}, z_{i}\right) \\ 0 & \text { otherwise }\end{cases}
$$

The set $\left\{\mu_{i}-\mu_{i+1}\right\}_{i=1}^{N_{1}-1}$ is a basis of $H_{h}$. Therefore, to compute an approximation $b_{h}\left(\xi_{h}, \eta_{h}\right)$ of $b\left(\xi_{h}, \eta_{h}\right)$, for $\xi_{h}, \eta_{h} \in H_{h}$, it suffices to define a quadrature scheme to approximate the coefficients

$$
b_{i, j}:=b\left(\mu_{i}, \mu_{j}\right)=-\frac{1}{4 \pi} \int_{z_{i-1}}^{z_{i}} \int_{z_{j-1}}^{z_{j}} \log |\boldsymbol{x}(s)-\boldsymbol{x}(t)|^{2} d s d t
$$

for $i, j=1, \ldots, N_{1}$. Due to the periodicity of $\boldsymbol{x}$, the coefficients $b_{i, j}$ can be defined for any pair of integer numbers $(i, j)$ modulo $N_{1}$. We define a scheme to approximate the coefficients corresponding to indices $(i, j)$ in the set $\mathcal{I}:=\left\{(i, j) \in \mathbb{Z} \times \mathbb{Z} ;|i-j| \leq N_{1} / 2\right\}$. Then, for $(i, j) \in \mathcal{I}$, we make the decomposition:

$$
b_{i, j}=-\frac{1}{4 \pi}\left(\int_{z_{i-1}}^{z_{i}} \int_{z_{j-1}}^{z_{j}} F(s, t) d s d t+\int_{z_{i-1}}^{z_{i}} \int_{z_{j-1}}^{z_{j}} \log (s-t)^{2} d s d t\right)
$$

where

$$
F(s, t):= \begin{cases}\log \frac{|\boldsymbol{x}(s)-\boldsymbol{x}(t)|^{2}}{(s-t)^{2}} & \text { si } s \neq t \\ \log \left|\boldsymbol{x}^{\prime}(s)\right|^{2} & \text { si } s=t\end{cases}
$$


We remark that $F$ is a function of class $\mathcal{C}^{\infty}$ in the set $\left\{(s, t) \in \mathbb{R}^{2} ;|s-t|<1\right\}$. Therefore, if $(i, j) \in \mathcal{I}$, the first term of the decomposition can be approximated using $\hat{\ell}_{2}$. On the other hand, elementary calculations give that

$$
\int_{z_{i-1}}^{z_{i}} \int_{z_{j-1}}^{z_{j}} \log (s-t)^{2} d s d t=h^{2}\left(\log h^{2}+B_{i-j}\right)
$$

where for every $k \in \mathbb{Z}, B_{k}:=\int_{0}^{1} \int_{0}^{1} \log (k+s-t)^{2} d s d t$. The constants $B_{k}$ are independent of the curve $\Gamma$ and can be calculated exactly. However, its exact evaluation is unstable since in its expression appear differences of large quantities. They are usually approximated as in [11]. We remark that $B_{k}=B_{-k}$, for all $k \in \mathbb{Z}$. Therefore, for $i, j=1, \ldots, N_{1}$, we calculate

$$
\tilde{b}_{i, j}:=-\frac{1}{4 \pi} h^{2}\left(\hat{\ell}_{2}\left(F\left(z_{\underline{i}-1}+h \cdot, z_{\underline{j}-1}+h \cdot\right)\right)+\log h^{2}+B_{\underline{i}-\underline{j}}\right)
$$

where

$$
(\underline{i}, \underline{j}):= \begin{cases}(i, j) & \text { si }(i, j) \in \mathcal{I} \\ \left(i, j+N_{1}\right) & \text { si } i-j>N_{1} / 2 \\ \left(i, j-N_{1}\right) & \text { si } j-i>N_{1} / 2\end{cases}
$$

Then, the approximate bilinear form $b_{h}: H_{h} \times H_{h} \rightarrow \mathbb{R}$ is given by

$$
b_{h}\left(\xi_{h}, \eta_{h}\right):=\sum_{i, j=1}^{N_{1}} \xi_{i} \eta_{j} \tilde{b}_{i, j} \quad \forall \xi_{h}, \eta_{h} \in H_{h}
$$

We recall that for $\eta_{h} \in H_{h}, \eta_{i}$ denotes the constant value of $\eta_{h}$ on $\left(z_{i-1}, z_{i}\right)$.

Now, to define an approximation of the bilinear form $d(\cdot, \cdot)$ in $V_{h} \times V_{h}$, we remark that if $v_{h} \in V_{h}$, then $\gamma\left(v_{h}\right) \in T_{h}$, where

$$
T_{h}:=\left\{\eta_{h} \in \mathcal{C}(\mathbb{R}) ; \eta_{h}(s)=\eta_{h}(s+1) \quad \forall s \in \mathbb{R} \quad \text { and }\left.\quad \eta_{h}\right|_{\left(z_{i-1}, z_{i}\right)} \in \mathcal{P}_{1} \quad \forall i \in \mathbb{Z}\right\}
$$

Let $\left\{\ell_{i}\right\}_{i=1}^{N_{1}}$ be the nodal basis of $T_{h}$. Then, it suffices to define a scheme to approximate

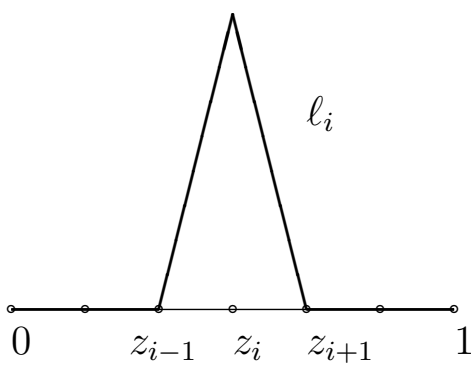

Figure 3: Functions $\ell_{i}$

the coefficients $d_{i, j}:=b\left(\ell_{i}^{\prime}, \ell_{j}^{\prime}\right)=\frac{1}{h^{2}}\left(b_{i, j}-b_{i, j+1}-b_{i+1, j}+b_{i+1, j+1}\right)$, for $i, j=1, \ldots, N_{1}$. If we call

$$
\tilde{d}_{i, j}:=\frac{1}{h^{2}}\left(\tilde{b}_{i, j}-\tilde{b}_{i, j+1}-\tilde{b}_{i+1, j}+\tilde{b}_{i+1, j+1}\right) \quad i, j=1, \ldots, N_{1}
$$


then the approximate bilinear form $d_{h}: V_{h} \times V_{h} \rightarrow \mathbb{R}$ is given by

$$
d_{h}\left(u_{h}, v_{h}\right):=\sum_{i, j=1}^{N_{1}} \gamma u\left(z_{i}\right) \gamma v\left(z_{j}\right) \tilde{d}_{i, j} \quad \forall u_{h}, v_{h} \in V_{h}
$$

Finally, we define an approximation of the bilinear form $c(\cdot, \cdot)$ in $V_{h} \times H_{h}$. Let $v_{h} \in V_{h}$ and $\eta_{h} \in H_{h}$. Then, we calculate $\left(\eta_{h}, \gamma\left(v_{h}\right)\right)$ exactly. So, we only have to define a scheme to approximate the coefficients

$$
c_{i, j}:=\int_{z_{j-1}}^{z_{j}}\left(\int_{z_{i-1}}^{z_{i+1}} K(s, t) l_{i}(t) d t\right) d s \quad i, j=1, \ldots, N_{1}
$$

Since the kernel $K(\cdot, \cdot)$ of $\mathcal{K}$ is a function of class $\mathcal{C}^{\infty}$, we use $\hat{\ell}_{2}$ to define the approximations

$$
\tilde{c}_{i, j}:=h^{2} \hat{\ell}_{2}\left(K\left(z_{j-1}+h \cdot, z_{i-1}+h \cdot\right) l_{i}\left(z_{i-1}+h \cdot\right)+K\left(z_{j-1}+h \cdot, z_{i}+h \cdot\right) l_{i}\left(z_{i}+h \cdot\right)\right)
$$

Therefore, we define the bilinear form $c_{h}: V_{h} \times H_{h} \rightarrow \mathbb{R}$ by

$$
c_{h}\left(v_{h}, \eta_{h}\right):=\frac{h}{4} \sum_{j=1}^{N_{1}} \eta_{j}\left(\gamma v\left(z_{j-1}\right)+\gamma v\left(z_{j}\right)\right)-\sum_{i, j=1}^{N_{1}} \eta_{j} \gamma v\left(z_{i}\right) \tilde{c}_{i, j}
$$

We approximate the form $A(\cdot, \cdot)$ by $A_{h}: M_{h} \times M_{h} \rightarrow \mathbb{R}$ defined by

$$
A_{h}\left(\hat{u}_{h}, \hat{v}_{h}\right):=a_{h}\left(u_{h}, v_{h}\right)+B_{h}\left(\hat{u}_{h}, \hat{v}_{h}\right) \quad \forall \hat{u}_{h}, \hat{v}_{h} \in M_{h}
$$

where

$$
B_{h}\left(\hat{u}_{h}, \hat{v}_{h}\right):=d_{h}\left(u_{h}, v_{h}\right)-c_{h}\left(v_{h}, \xi_{h}\right)+c_{h}\left(u_{h}, \eta_{h}\right)+b_{h}\left(\xi_{h}, \eta_{h}\right)
$$

Then, we propose the following scheme to approximate the solution to problem (12): Find $\hat{u}_{h}^{n}:=\left(u_{h}^{n}, \xi_{h}^{n}\right) \in M_{h}\left(n=1, \ldots, N_{0}\right)$ such that

$$
\left(u_{h}^{n}-u_{h}^{n-1}, v_{h}\right)_{L^{2}\left(\Omega_{P}\right)}+\Delta t A_{h}\left(\hat{u}_{h}^{n}, \hat{v}_{h}\right)=\Delta t\left(f\left(t_{n}\right), v_{h}\right)_{h, P} \quad \forall \hat{v}_{h} \in M_{h}
$$

where $u_{h}^{0}:=u_{0, h} \in \mathcal{C}\left(\bar{\Omega}_{P}\right)$ is such that $\left(u_{h}^{0}\right)_{\mid T} \in \mathcal{P}_{1}(T) \forall T \in \tau_{h, P}$ and $u_{h}^{0} \rightarrow u_{0}$ in $L^{2}\left(\Omega_{P}\right)$. Here, we suppose that the terms $\left(u_{h}, v_{h}\right)_{L^{2}\left(\Omega_{P}\right)}$, with $u_{h}, v_{h} \in V_{h}$, are computed exactly since the triangulation $\tau_{h, P}$ only contains straight triangles. Nevertheless, in practice it is usual to apply the lumped masses method (cf. V. Thomée [21]).

Lemma 9. There exists a constant $C>0$, independent of $h$, such that

$$
\left|B\left(\hat{u}_{h}, \hat{v}_{h}\right)-B_{h}\left(\hat{u}_{h}, \hat{v}_{h}\right)\right| \leq C h\left\|\hat{u}_{h}\right\|_{M}\left\|\hat{v}_{h}\right\|_{M} \quad \forall \hat{u}_{h}, \hat{v}_{h} \in M_{h}
$$

Proof. In Lemma 11 of [7], it is proved that there exists a constant $C>0$, independent of $h$, such that

$$
\left|b\left(\xi_{h}, \eta_{h}\right)-b_{h}\left(\xi_{h}, \eta_{h}\right)\right| \leq C h\left\|\xi_{h}\right\|_{-1 / 2}\left\|\eta_{h}\right\|_{-1 / 2} \quad \forall \xi_{h}, \eta_{h} \in H_{h}
$$


On the other hand, since operators $\frac{d}{d s}: H^{1 / 2} \rightarrow H_{0}^{-1 / 2}$ and $\gamma: H^{1}(\Omega) \rightarrow H^{1 / 2}$ are continuous, we deduce that there exists a constant $C>0$, independent of $h$, such that

$$
\left|d\left(u_{h}, v_{h}\right)-d_{h}\left(u_{h}, v_{h}\right)\right| \leq C h\left\|u_{h}\right\|_{1, \Omega^{-}}\left\|v_{h}\right\|_{1, \Omega^{-}} \quad \forall u_{h}, v_{h} \in V_{h}
$$

Lastly, in Lemma 5.2 of [18] it is proved that there exists a constant $C>0$, independent of $h$, such that

$$
\left|c\left(v_{h}, \eta_{h}\right)-c_{h}\left(v_{h}, \eta_{h}\right)\right| \leq C h^{3 / 2}\left\|v_{h}\right\|_{1, \Omega^{-}}\left\|\eta_{h}\right\|_{-1 / 2} \quad \forall v_{h} \in V_{h} \quad \forall \eta_{h} \in H_{h}
$$

The result follows using the triangular inequality.

Theorem 10. Assume that $\mathbf{b}$ satisfies conditions (5) and (10). Then problem (25) has a unique solution, for $h$ sufficiently small.

Proof. In Theorem 35.2 of [22], it is proved that under conditions (5) and (10), the forms $a_{h}(\cdot, \cdot)$ are uniformly strongly monotone and uniformly bounded in $V_{h}$, for some $h \leq h_{0}$. On the other hand, Lemma 9 implies that the bilinear forms $B_{h}(\cdot, \cdot)$ verify these properties as well, if $h$ is sufficiently small. Therefore, if $\mathbf{b}$ satisfies (5) and (10), the forms $A_{h}(\cdot, \cdot)$ are uniformly strongly monotone and uniformly bounded in $M_{h}$, for $h \leq h_{0}$. The rest of the proof is analogous to that of Theorem 3 .

To study the effect of quadratures on convergence, we will use the following auxiliary lemma, which is proved in [22, Lema 26.7].

Lemma 11. Let $T \in \tau_{h}$ and suppose that $f \in W^{1, \infty}(T)$. Then there exists a constant $C>0$, independent of $T$ and $f$, such that

$$
\left|\int_{T} f p d \mathbf{x}-Q_{T}(f p)\right| \leq C h_{T} m e s(T)^{1 / 2}\|f\|_{W^{1, \infty}(T)}\|p\|_{H^{1}(T)} \quad \forall p \in \mathcal{P}_{1}(T)
$$

where $h_{T}$ denotes the diameter of triangle $T$.

We also have to impose additional conditions on $\mathbf{b}$. In the rest of the paper, we suppose that, besides (5), (10) and (11), the derivatives $\frac{\partial b_{i}}{\partial x_{j}}: \bar{\Omega}_{P} \times \mathbb{R}^{3} \rightarrow \mathbb{R}(i, j=1,2)$ are continuous in $\bar{\Omega}_{P} \times \mathbb{R}^{3}$ and there exists a constant $C>0$ such that

$$
\left|\frac{\partial b_{i}}{\partial x_{j}}(\mathbf{x}, \boldsymbol{\alpha})\right| \leq C(1+|\boldsymbol{\alpha}|) \quad \forall(\mathbf{x}, \boldsymbol{\alpha}) \in \bar{\Omega}_{P} \times \mathbb{R}^{3}
$$

Lemma 12. If conditions (5), (11) and (26) are satisfied, then there exists a constant $C>0$, independent of $h$, such that

$$
\left|A\left(\hat{u}_{h}, \hat{v}_{h}\right)-A_{h}\left(\hat{u}_{h}, \hat{v}_{h}\right)\right| \leq C h\left(1+\left\|\hat{u}_{h}\right\|_{M}\right)\left\|\hat{v}_{h}\right\|_{M} \quad \forall \hat{u}_{h}, \hat{v}_{h} \in M_{h}
$$

Proof. In these conditions, M. Feistauer [8] proved that there exists a constant $C>0$, independent of $h$, such that

$$
\left|a\left(u_{h}, v_{h}\right)-a_{h}\left(u_{h}, v_{h}\right)\right| \leq C h\left(1+\left\|u_{h}\right\|_{H^{1}(\Omega)}\right)\left\|v_{h}\right\|_{H^{1}(\Omega)} \quad \forall u_{h}, v_{h} \in V_{h}
$$

The result follows combining this inequality with Lemma 9. 
Theorem 13. Under the assumptions of Theorem 6, if moreover $\mathbf{b}$ satisfies (26) and $f \in \mathcal{C}\left([0, T] ; W^{1, \infty}\left(\Omega_{P}\right)\right)$, the sequence $\left(\hat{u}_{h, e}\right)_{h}$ converges in $L^{2}(0, T ; M)$ to the solution to problem (9).

Proof. Proceeding similarly to Lemma 4, we can prove a priori error estimates for the solution to the fully discrete scheme (25). In effect, if conditions (5) and (10) are satisfied and $\Delta t$ is sufficiently small, there exists a constant $C>0$, independent of $h$ and $\Delta t$, such that

$$
\left\|u_{h, e}(T)\right\|_{L^{2}\left(\Omega_{P}\right)}+\left\|\hat{u}_{h, e}\right\|_{L^{2}(0, T ; M)} \leq C
$$

Therefore, it is possible to extract weakly convergent subsequences $\left(u_{h^{\prime}, e}(T)\right)_{h^{\prime}} \rightarrow g^{*}$ in $L^{2}\left(\Omega_{P}\right)$ and $\left(\hat{u}_{h^{\prime}, e}\right)_{h^{\prime}} \rightarrow \hat{u}^{*}$ in $L^{2}(0, T ; M)$. Now, let $\phi \in \mathcal{C}^{\infty}([0, T])$. Given $\hat{w} \in M$, we consider a sequence $\left(\hat{w}_{h}\right)_{h}, \hat{w}_{h} \in M_{h}$, converging to $\hat{w}$ in $M$. Putting $\hat{v}_{h}=\hat{w}_{h} \phi\left(t_{n}\right)$ in equation (25) and summing by parts, we obtain that

$$
\begin{gathered}
\int_{0}^{T}\left(f_{e}(t), w_{h}\right)_{h, P} \phi_{e}(t) d t=\left(u_{h, e}(T), w_{h}\right)_{L^{2}\left(\Omega_{P}\right)} \phi(T)-\left(u_{0, h}, w_{h}\right)_{L^{2}\left(\Omega_{P}\right)} \phi(\Delta t) \\
+\int_{0}^{T}\left(u_{h, e}(t), w_{h}\right)_{L^{2}\left(\Omega_{P}\right)} \tilde{\phi}_{e}(t) d t-\int_{0}^{T} A_{h}\left(\hat{u}_{h, e}(t), \hat{w}_{h}\right) \phi_{e}(t) d t
\end{gathered}
$$

For each $h^{\prime}$ and every $t \in[0, T]$, we consider the functional $\chi_{h^{\prime}}^{*}(t) \in M^{\prime}$ defined by

$$
\left\langle\chi_{h^{\prime}}^{*}(t), \hat{v}\right\rangle:=A\left(\hat{u}_{h^{\prime}, e}(t), \hat{v}\right) \quad \forall \hat{v} \in M
$$

As in the proof of Theorem 6 , we can extract a subsequence of $\left(\chi_{h^{\prime}}^{*}\right)_{h^{\prime}}$ (that we will denote the same) converging weakly to an element $\chi^{*} \in L^{2}\left(0, T ; M^{\prime}\right)$.

Applying Lemma 12 and the Cauchy-Schwarz inequality, we deduce that

$$
\int_{0}^{T}\left(A_{h^{\prime}}\left(\hat{u}_{h^{\prime}, e}(t), \hat{w}_{h^{\prime}}\right)-A\left(\hat{u}_{h^{\prime}, e}(t), \hat{w}_{h^{\prime}}\right)\right) \phi_{e}(t) d t \longrightarrow 0 \quad \text { as } h^{\prime} \rightarrow 0
$$

Similarly, by virtue of Lemma 11 we have that

$$
\int_{0}^{T}\left(\left(f_{e}(t), w_{h^{\prime}}\right)_{h, P}-\left(f_{e}(t), w_{h^{\prime}}\right)_{L^{2}\left(\Omega_{P}\right)}\right) \phi_{e}(t) d t \longrightarrow 0 \quad \text { as } h^{\prime} \rightarrow 0
$$

Thus, passing to the limit as $h^{\prime} \rightarrow 0$ in (27), we obtain

$$
\begin{aligned}
& \left(g^{*}, w\right)_{L^{2}\left(\Omega_{P}\right)} \phi(T)-\left(u_{0}, w\right)_{L^{2}\left(\Omega_{P}\right)} \phi(0)-\int_{0}^{T}\left(u^{*}(t), w\right)_{L^{2}\left(\Omega_{P}\right)} \phi^{\prime}(t) d t \\
& +\int_{0}^{T}\left\langle\chi^{*}(t), \hat{w}\right\rangle \phi(t) d t=\int_{0}^{T}(f(t), w)_{L^{2}\left(\Omega_{P}\right)} \phi(t) d t
\end{aligned}
$$

Following the proof of Theorem 6 , we deduce that $\hat{u}^{*}=\hat{u}$ is the solution to problem (9) and that the sequence $\left(\hat{u}_{h, e}\right)_{h}$ converges to $\hat{u}$ in $L^{2}(0, T ; M)$.

Finally, we prove that the order of convergence of the solutions to the fully discrete scheme (25) remains optimal. 
Theorem 14. Under the hypothesis of Theorems 8 and 13, there exists a constant $C>0$, independent of $h$ and $\Delta t$, such that

$$
\left\|\hat{u}_{e}-\hat{u}_{h, e}\right\|_{L^{2}(0, T ; M)} \leq C\left(h^{\sigma}+\Delta t\right)
$$

Proof. For $n=1, \ldots, N_{0}$, we denote by $\hat{e}_{h}^{n}:=\hat{u}_{h}^{n}-\widehat{\Pi}_{h} \hat{u}\left(t_{n}\right)$ and $\tilde{u}_{h}^{n}:=\Pi_{h} u\left(t_{n}\right)$. Let $e_{h}^{0}:=0$ and $\tilde{u}_{h}^{0}:=u_{0, h}$. We recall that $\left(\hat{u}_{h}^{n}\right)_{n=1}^{N_{0}}$ is the solution to scheme (25) and that under the hypothesis of the theorem, $\hat{u}$ satisfies equation (9) for all $t \in(0, T]$. Then, we deduce that

$$
\begin{gathered}
\left(e_{h}^{n}-e_{h}^{n-1}, v_{h}\right)_{L^{2}\left(\Omega_{P}\right)}+\Delta t\left(A_{h}\left(\hat{u}_{h}^{n}, \hat{v}_{h}\right)-A_{h}\left(\widehat{\Pi}_{h} \hat{u}\left(t_{n}\right), \hat{v}_{h}\right)\right) \\
=\left(\Delta t u^{\prime}\left(t_{n}\right)-\left(u\left(t_{n}\right)-u\left(t_{n-1}\right)\right), v_{h}\right)_{L^{2}\left(\Omega_{P}\right)}+\Delta t\left(A\left(\hat{u}\left(t_{n}\right), \hat{v}_{h}\right)-A\left(\widehat{\Pi}_{h} \hat{u}\left(t_{n}\right), \hat{v}_{h}\right)\right) \\
+\left(u\left(t_{n}\right)-u\left(t_{n-1}\right)-\left(\tilde{u}_{h}^{n}-\tilde{u}_{h}^{n-1}\right), v_{h}\right)_{L^{2}\left(\Omega_{P}\right)}+\Delta t\left(A\left(\widehat{\Pi}_{h} \hat{u}\left(t_{n}\right), \hat{v}_{h}\right)-A_{h}\left(\widehat{\Pi}_{h} \hat{u}\left(t_{n}\right), \hat{v}_{h}\right)\right) \\
+\Delta t\left(\left(f\left(t_{n}\right), v_{h}\right)_{h, P}-\left(f\left(t_{n}\right), v_{h}\right)_{L^{2}\left(\Omega_{P}\right)}\right)
\end{gathered}
$$

Using Lemma 12 and the continuity of $\widehat{\Pi}_{h}: M \rightarrow M_{h}$, we deduce that

$$
\left|\left(A\left(\widehat{\Pi}_{h} \hat{u}\left(t_{n}\right), \hat{v}_{h}\right)-A_{h}\left(\widehat{\Pi}_{h} \hat{u}\left(t_{n}\right), \hat{v}_{h}\right)\right)\right| \leq C h\left\|\hat{u}\left(t_{n}\right)\right\|_{M}\left\|\hat{v}_{h}\right\|_{M}
$$

On the other hand, applying Lemma 11, we have that

$$
\left|\left(f\left(t_{n}\right), v_{h}\right)_{h, P}-\left(f\left(t_{n}\right), v_{h}\right)_{L^{2}\left(\Omega_{P}\right)}\right| \leq C h\|f\|_{\mathcal{C}\left([0, T] ; W^{1, \infty}\left(\Omega_{P}\right)\right.}\left\|\hat{v}_{h}\right\|_{M}
$$

The rest of the proof is analogous to that of Theorem 8 .

\section{References}

[1] C. Bernardi, Optimal finite element interpolation on curved domains, SIAM J. Numer. Anal. 26 (1989) 1212-1240.

[2] C. Carstensen, S.A. Funken and E.P. Stephan, On the adaptive coupling of FEM and BEM in 2-d-elasticity, Numer. Math. 77 (1997) 187-221.

[3] Ph. Clément, Approximation by finite element functions using local regularization, RAIRO 9 (1975) 77-84.

[4] M. Costabel, A symmetric method for the coupling of finite elements and boundary elements, in: J.R. Whiteman. (Ed.), The Mathematics of Finite Elements and Applications, Vol. IV, Academic Press, London, 1988, pp. 281-288.

[5] M. Costabel, V.J. Ervin and E.P. Stephan, Symmetric coupling of finite elements and boundary elements for a parabolic-elliptic interface problem, Quart. J. Appl. Math. 48 (1990) 265-279.

[6] M. Costabel and E.P. Stephan, Coupling of finite and boundary element methods for an elastoplastic interface problem, SIAM J. Numer. Anal. 27 (1990) 1212-1226. 
[7] M. Crouzeix and F.J. Sayas, Asymptotic expansions of the error of spline Galerkin boundary element methods, Numer. Math. 78 (1998) 523-547.

[8] M. Feistauer, On the finite element approximation of a cascade flow problem, Numer. Math. 50 (1987) 655-684.

[9] G.N. Gatica and G.C. Hsiao, Boundary-field equation methods for a class of nonlinear problems, Pitman Research Notes in Math. Series 331, Longman, 1995.

[10] H.D. Han, A new class of variational formulations for the coupling of finite and boundary element methods, J. Comp. Math. 8 (1990) 223-232.

[11] G.C. Hsiao, P. Kopp and W.L. Wendland, A Galerkin collocation method for some integral equations of the first kind, Computing 25 (1979) 557-566.

[12] C. Johnson and J.C. Nédélec, On the coupling of boundary integral and finite element methods, Math. Comp. 35 (1980) 1063-1079.

[13] R. Kress, Linear Integral Equations, Applied Mathematical Sciences 82, SpringerVerlag (1999).

[14] A. Kufner, O. John and S. Fučík, Function spaces, Academia, Praga, 1977.

[15] J.L. Lions and E. Magenes, Problèmes aux limites non homogènes et applications, Vol. I, Dunod, Paris, 1969.

[16] R.C. MacCamy and M. Suri, A time-dependent interface problem for twodimensional eddy currents, Quart. Appl. Math. 44 (1987) 675-690.

[17] S. Meddahi and M. González, A fully discrete BEM-FEM method for an exterior elasticity system in the plane, J. Comput. Appl. Maths 134 (2001) 127-141.

[18] S. Meddahi, M. González and P. Pérez, On a FEM-BEM formulation for an exterior quasilinear problem in the plane, SIAM J. Numer. Anal. 37 (2000) 1820-1837.

[19] P. Mund and E.P. Stephan, Adaptive coupling and fast solution of FEM-BEM equations for parabolic-elliptic interface problems, Math. Meth. Appl. Sci. 20 (1997) 403-423.

[20] J.T. Oden, Qualitative methods in nonlinear mechanics, Prentice-Hall, 1986.

[21] V. Thomée, Galerkin finite element methods for parabolic problems, Springer Series in Computational Mathematics 25, Springer-Verlag, 1997.

[22] A. Ženíšek, Nonlinear elliptic and evolution problems and their finite element approximations, Computational Mathematics and Applications, Academic Press, London, 1990.

[23] M. Zlámal, Curved elements in the finite element method I, SIAM J. Numer. Anal. 10 (1973) 229-240. 
[24] M. Zlámal, Curved elements in the finite element method II, SIAM J. Numer. Anal. 11 (1974) 347-362.

[25] M. Zlámal, Finite element solution of quasistationary nonlinear magnetic field, RAIRO Anal. Numér. 16 (1982) 161-191. Addendum, RAIRO Anal. Numér. 17 (1983) 407-415. 